\title{
TV/Series
}

6 | 2014

Écho et reprise dans les séries télévisées (III) : de la métafiction à la transmédialité

\section{L'art du teaser : les séquences prégénériques dans quelques séries fantastiques américaines des années 1990 et 2000}

\section{Claire Cornillon}

\section{OpenEdition}

\section{Journals}

Édition électronique

URL : http://journals.openedition.org/tvseries/314

DOI : 10.4000/tvseries.314

ISSN : 2266-0909

Éditeur

GRIC - Groupe de recherche Identités et Cultures

\section{Référence électronique}

Claire Cornillon, «L'art du teaser : les séquences prégénériques dans quelques séries fantastiques américaines des années 1990 et 2000 », TV/Series [En ligne], 6 | 2014, mis en ligne le 01 décembre 2014, consulté le 20 avril 2019. URL : http://journals.openedition.org/tvseries/314 ; DOI : 10.4000/ tvseries.314

\section{c) (i)}

TV/Series est mis à disposition selon les termes de la licence Creative Commons Attribution - Pas d'Utilisation Commerciale - Pas de Modification 4.0 International. 


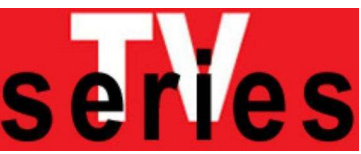

\section{L'art du teaser : les séquences prégénériques dans quelques séries fantastiques américaines des années 1990 et 2000}

Claire CORNILLON

Le teaser ou séquence prégénérique est une lieu clef de la sérialité. Placé avant le générique, il constitue à la fois une entrée dans la fiction, un retour à un monde fictionnel que l'on connaît déjà et une ouverture vers quelque chose d'inédit. Jouant sur les codes, et les effets de reprises, il se construit à la fois comme entité autonome et comme moment d'articulation entre un avant et un après, tant à l'échelle de l'épisode qu'à l'échelle de la série. Les séries télévisées des années 1990-2000 ont particulièrement travaillé cette forme pour en tirer toutes les possibilités narratives, structurelles ou esthétiques. Les séries de genre, et notamment les séries fantastiques, y trouvent un lieu propice à l'élaboration de leurs propoes codes génériques. L'article se propose donc d'analyser quelques cas de séquences prégénériques, dans le cadre de séries fantastiques américaines des années 19902000.

$\mathrm{L}$ a série télévisée est une œuvre qui se construit autour d'un format et de contraintes financières, structurelles ou narratives souvent bien délimitées. Depuis le début des années 1990, les séries télévisées américaines connaissent de fortes mutations en termes de structures, de narration et d'esthétique. Au modèle qui s'était imposé jusqu'alors, dans lequel chaque épisode était une variation autonome sur une structure similaire, se substitue un modèle semifeuilletonnant. La terminologie dans ce domaine distingue en effet « la série ", qui propose une suite d'épisodes mettant en scène des personnages récurrents mais qui constituent des unités relativement indépendantes, et «le feuilleton », dont l'intrigue unique se poursuit d'épisode en épisode. Dans le modèle qui s'impose au cours des années 1990, si chaque épisode raconte bien une histoire close, la saison, voire la série dans son ensemble, développent une intrigue, un arc narratif, qui se poursuit de semaine en semaine. Stéphane Benassi le constatait en ces termes dans son ouvrage paru à cette même période :

Depuis quelques temps, nous assistons en effet à l'émergence de fictions qui semblent tendre vers un compromis presque « parfait » entre la série et le feuilleton. Ainsi, certaines fictions sérielles, telles que Code Quantum ou Slider, sont-elles constituées d'épisodes narrant les aventures de personnages récurrents et possédant une unité diégétique propre, bien que la fin de chacun de ces épisodes annonce le début de l'épisode suivant, créant l'attente chez le spectateur comme le ferait un feuilleton. D'autres fictions, quant à elles, apparaissent comme étant des feuilletons dont chaque épisode possède une unité diégétique propre. C'est le cas, par exemples, d'Urgences ou de New York Police Blues. Bien que ces compromis entre série et feuilleton ne soient pas nouveaux et remontent aux années 
soixante, il semble intéressant d'observer de quelles façons ils tendent aujourd'hui à se diversifier et à se complexifier ${ }^{1}$.

Le format de la fiction sérielle connaît donc des mutations qui relèvent d'une forme d'hybridation entre la série et le feuilleton. L'approche du personnage s'en trouve dès lors modifiée, comme l'ajoute par ailleurs Stéphane Benassi : " Le héros de la série classique a également ceci de particulier qu'il ne subit aucune évolution au fil des épisodes $[\ldots]^{2}$. » En revanche, dans les séries des années 1990 et 2000, l'évolution du personnage devient un des enjeux majeurs de la narration au point que celui-ci en vient à structurer la série, assurant précisément la continuité de celle-ci au-delà de l'autonomie des épisodes.

Par ailleurs, le format de l'épisode lui-même entretient une ambivalence similaire entre l'autonomie et la continuité. Chacun reprend en effet la même structure, qui s'impose dans cette décennie 1990: le résumé des épisodes précédents ${ }^{3}$, puis la séquence prégénérique, précèdent le générique lui-même et le corps de l'épisode. Ce teaser ${ }^{4}$, ou séquence prégénérique, devient le lieu fondamental qui doit happer le téléspectateur pour éviter qu'il ne zappe ; il se construit donc entre deux éléments récurrents plus ou moins fixes, et se doit à la fois d'introduire l'épisode et de laisser des éléments en suspens afin de susciter la curiosité. Plus encore, la séquence prégénérique est le seuil qui porte l'identité de la série, puisqu'elle plonge le spectateur dans les codes d'un genre, dans une certaine spécificité de l'image, de la mise en scène et de la musique, tout en renouvelant constamment l'intérêt du spectateur. Dès lors, cette séquence se construit sur une dialectique entre répétition et nouveauté et oscille entre, d'un côté, une continuité très forte avec le reste de la série et le corps de l'épisode lui-même et, de l'autre, une autonomie relative par rapport à l'épisode qu'il introduit et à l'ensemble de la série. Si certaines séries choisissent de centrer le teaser sur des personnages secondaires, d'autres mettent en scène les héros de l'intrigue dès les premières images ; d'aucunes installent, dès cette séquence, le sujet de l'épisode quand d'autres y voient l'occasion d'une digression fertile. Les stratégies sont multiples, d'une série à l'autre, mais aussi d'un épisode à l'autre.

${ }^{1}$ Stéphane Benassi, Séries et feuilletons T.V. Pour une typologie des fictions télévisuelles, Liège, Editions du CEFAL, 200o, p. 37

${ }^{2}$ Ibid., p. 84 .

3 Le résumé des épisodes précédents est parfois remplacé par une brève présentation de la série que l'on retrouve au début de chaque épisode et qui est destinée elle aussi à exposer le contexte au téléspectateur néophyte - mais ceci dans le cadre de la série en général et non pas seulement des épisodes qui précèdent. C'est le cas, par exemple, dans la première saison de Buffy The Vampire Slayer.

${ }^{4}$ Le terme «teaser » a deux sens. Il peut désigner une pré-bande annonce [c'est-à-dire une bande annonce très courte]. Ici, nous l'employons au sens de "séquence prégénérique » [c'est-à-dire une séquence de l'épisode qui se situe avant le générique]. 
Ce modèle, s'il persiste encore aujourd'hui 5 , et pas seulement dans la fiction américaine ${ }^{6}$, est concurrencé depuis le début des années 2000 par d'autres tendances: la vogue des séries entièrement feuilletonnantes comme 24 (Fox, 2001-2010) offre d'autres stratégies narratives7. Par ailleurs, certaines séries ne proposent plus de teaser (c'est le cas de nombreuses séries HBO), alors que le générique luimême a eu tendance, dans certains cas, à disparaitre au profit d'un simple logo, comme dans Supernatural ${ }^{8}$. Ces nouvelles formules travaillent le format de la série d'une autre manière, tout en s'appuyant sur les expériences narratives qui les ont précédées. La tendance actuelle n'est plus à l'unicité mais à la pluralité. Dans l'abondance de l'offre, toutes les stratégies peuvent cohabiter, et les créateurs usent désormais des normes comme ils le souhaitent, s'inscrivant dans la tradition sur certains points et innovant sur d'autres, d'autant que la conscience même d'une historicité du genre amène les auteurs à revendiquer leur généalogie 9 .

Remarquons également que la séquence prégénérique n'est pas la prérogative des seules séries et qu'elle existe déjà au cinéma. On pense, parmi de nombreux exemples, à l'ouverture célèbre du film Kiss Me Deadly de Robert Aldrich (1955). Le film commence in medias res alors qu'une jeune femme court pieds nus sur une route la nuit. Elle arrête une voiture et son conducteur la prend en stop. L'ouverture plonge ainsi le spectateur dans l'action et le suspense par le biais d'une séquence mystérieuse qui vient s'imposer avant même que les crédits du film n'apparaissent. On peut d'ailleurs faire l'hypothèse d'une influence réciproque des deux arts sur ce sujet. Alexandre Tylski souligne ce phénomène dans le cinéma des années 1960 :

Mais une autre technique de fragmentation des génériques est également employée à cette époque, les pré-génériques - qui pourraient aisément faire l'objet d'un livre entier. Leur emploi dans les épisodes de La Panthère rose et autres épisodes de James Bond reste célèbre et témoigne souvent d'une volonté de

\footnotetext{
${ }^{5}$ Supernatural (The WB, 2005-) en est un exemple parmi d'autres.

${ }^{6}$ The Fades (BBC Three, 2011) exploite les mêmes principes.

7 Dans le cas d'une série entièrement feuilletonnante, le rapport entre la séquence prégénérique et le reste de l'épisode ou l'épisode précédent est différent puisqu'il est déterminé par la chronologie des événements.

${ }^{8}$ Sur la question du générique, voir Ariane Hudelet «Un cadavre ambulant, un petitdéjeuner sanglant, et le quartier Ouest de Baltimore : le générique, moment-clé de séries télévisées ", in Sarah Hatchuel et Monica Michlin (éds.), Les Pièges des nouvelles séries télévisées américaines : mécanismes narratifs et idéologiques, in GRAAT, No. 6, décembre 2009. http://www.graat.fr/backissuepiegesseriestv.htm, lien consulté le 11 décembre 2014. Voir aussi Eric Vérat, Génériques! Les séries américaines décryptées, Lyon, Les Moutons électriques, 2012.

${ }^{9}$ Bones (Fox, 2005- ) ou Fringe (Fox, 2008- ), par exemple, citent très rapidement The $X$ Files (Fox, 1993-2002), série fondatrice s'il en est, et il s'agit bien ici de clins d'œil « en hommage » plus que de formes d'allégeance à une série " maître ».
} 
stratégie narrative (et économique) motivée en grande partie par le succès des schémas télévisuels d'alors ${ }^{10}$.

Au-delà - ou peut-être en deçà - de ces remarques esthétiques, il est nécessaire de resituer tout discours sur les séries nordaméricaines dans un contexte qui est celui de la dimension économique de l'industrie télévisuelle des États-Unis. Les créateurs de ces œuvres travaillent dans un contexte extrêmement contraignant lié au support de diffusion (les exigences des networks ne sont, par exemple, pas les mêmes que celles des chaînes payantes du câble) mais aussi au problème des coupes publicitaires, de la censure, ou de l'autocensure, ainsi que des usages liés aux formats (le format court est ainsi généralement réservé aux sitcoms quand les séries dramatiques se déploient le plus souvent sur 40 à 50 minutes) et au nombre d'épisodes par saisons. Tous ces éléments influencent de manière très nette la création mais il est notable que les auteurs des œuvres que nous évoquerons usent de ces contraintes comme d'une source de créativité et les exploitent souvent de manière originale.

De fait, avec les séries fantastiques, dans lesquelles l'univers fictionnel est, par définition, le lieu de tous les possibles, la séquence d'ouverture d'un épisode est l'occasion pour le téléspectateur de découvrir également quelles en seront les règles cette semaine-là. C'est aussi l'occasion de jouer sur le statut fictionnel de l'image. Dans le teaser d'une série fantastique, puisque le contexte n'est pas encore fourni, et que l'on ne connait pas toujours avec certitude l'ancrage spatio-temporel, il serait tout à fait possible que nous soyons dans une dimension parallèle, un rêve ou une illusion quelconque. Les scénaristes vont jouer de cette possibilité en trompant le téléspectateur ou en cherchant à le surprendre, en brisant ses repères.

Cette analyse s'appuiera essentiellement sur des exemples tirés de The X-Files, de Chris Carter (Fox1993-2002), Buffy the Vampire Slayer, de Joss Whedon (WB, UPN 1997-2003), et Supernatural ${ }^{11}$, créée par Eric Kripke, (CW 2005-). L'article s'attachera à déterminer les fonctions de la séquence prégénérique dans ces trois séries, ainsi que son fonctionnement en termes structurels, narratifs et esthétiques avant de montrer comment cette séquence est une matrice, un lieu privilégié de la sérialité.

${ }^{10}$ Alexandre Tylski, Le Générique de cinéma. Histoire et fonctions d'un fragment hybride, Toulouse, Presses Universitaires du Mirail, 2008, p. 43.

${ }^{11}$ Série d'autant plus intéressante qu'elle s'inscrit dans une certaine tradition des années 1990, empruntant notamment à The $X$-Files, tout en adoptant des stratégies propres à des séries plus contemporaines. 


\section{Essai de typologie}

Pour établir une typologie des séquences prégénériques, quatre critères semblent essentiels : la question de la continuité avec l'épisode précédent, la présence des protagonistes de la série, le sujet de la séquence et sa durée. Ces critères posent les fondements du fonctionnement narratif de la séquence en interrogeant son lien avec l'épisode précédent et avec le corps de l'épisode auquel elle appartient. Ils permettent de comprendre comment la séquence pose son identité et son autonomie tout en s'articulant avec le reste de la série.

\section{La séquence prégénérique est-elle feuilletonnante ?}

Certains teasers reprennent l'intrigue exactement là où le cliffhanger de la fin de l'épisode précédent l'avait laissée. C'est le cas notamment dans les épisodes en deux parties, comme "What's my line » dans la saison 2 de Buffy the Vampire Slayer. La première partie se termine sur Kendra qui se présente à Buffy comme étant la Tueuse, et la seconde commence exactement après cette réplique, enchaînant simplement la suite de cette scène. Les exemples sont nombreux. Dans ce cas, le statut de la séquence prégénérique repose sur le fait qu'elle doit replonger le téléspectateur dans la réalité de la semaine d'avant : elle construit une continuité non pas seulement avec ce qui va suivre mais avec ce qui a précédé, ce qui relativise d'autant sa possible autonomie.

\section{Les personnages récurrents de la série sont-ils présents dans} la séquence prégénérique ?

The X-Files ou Supernatural mettent rarement en scène les protagonistes de la série dans les teasers. Lorsque cela arrive, l'épisode est précisément repérable comme un épisode particulièrement important parce qu'il se démarque de la norme présentée comme telle au spectateur. Ainsi, de nombreux épisodes de The $X$-Files, qui développent sa mythologie, c'est-à-dire son intrigue feuilletonnante, font apparaître Mulder ou Scully dès la séquence prégénérique, ce qui n'est pas la cas dans la plupart des autres épisodes. Dans ces deux séries fantastiques, dans lesquelles chaque épisode est une enquête sur un phénomène surnaturel, le prégénérique est le plus souvent le lieu de la présentation de la première victime, et le générique coïncide avec le moment d'apparition du surnaturel. L'apparition même du titre dans Supernatural, qui explose littéralement à l'écran, suivant des modalités qui varient de saison en saison, qualifie finalement ce qui vient d'être vu par le spectateur : un élément surnaturel (voir figure 1). Le générique, qui n'est qu'un logo, est une sorte d'étiquette qui agit rétroactivement sur la séquence prégénérique : ce que vous avez vu, il faut l'identifier comme un événement surnaturel et comme un événement qui appartient à l'univers de Supernatural. 


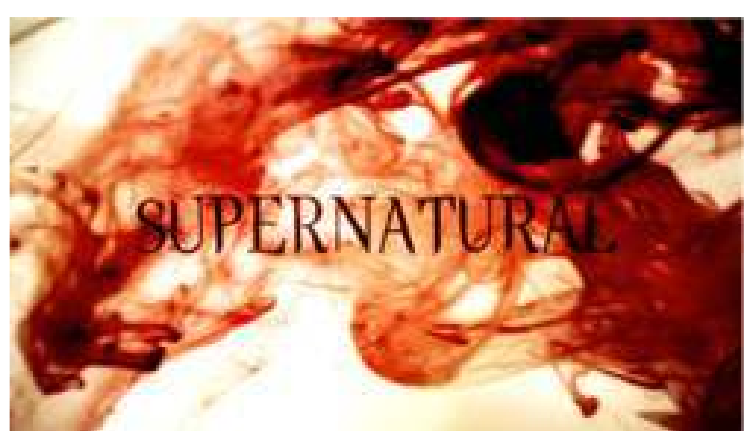

Fig. 1 : Générique de la saison 5 de Supernatural

Le principe est le même dans The $X$-Files. La plupart du temps, ce n'est que dans la scène qui suit le générique que les protagonistes de la série apparaissent et entament leur enquête. En revanche, Buffy the Vampire Slayer montre les protagonistes le plus souvent dès le prégénérique - précisément parce que le surnaturel est, dans cette série, plutôt un prétexte à l'évolution des personnages et davantage une métaphore d'expériences de la vie. Les personnages principaux et les relations qui se tissent entre eux constituent donc l'enjeu majeur de chacun des épisodes, faisant de l'intrigue autonome un catalyseur de leur évolution. La présence des protagonistes est donc essentielle dès la séquence prégénérique. Si les deux premières séries jouent souvent sur les mécanismes du film d'horreur, et présentent donc des premières victimes destinées à mourir de manière spectaculaire dans les séquences prégénériques, la dernière ne vise que très rarement à faire peur au téléspectateur. Ces diverses visées expliquent en partie les différences de stratégies narratives.

\section{Le teaser annonce-t-il le sujet de l'épisode ?}

La réponse à cette question n'est pas aussi évidente qu'on pourrait le croire car le prégénérique peut aussi proposer une ouverture que l'on peut qualifier d'anecdotique, c'est-à-dire qui ne fait pas avancer la narration et qui joue simplement le rôle d'une accroche. Par conséquent, l'isolement relatif du prégénérique peut être utilisé selon des objectifs différents: il peut, par exemple avoir une visée humoristique. Dans l'épisode «Teacher's pet » (4.1) de Buffy, qui se concentre sur la vie amoureuse de Xander, le prégénérique présente une scène qui se déroule au Bronze, bar bien connu des téléspectateurs, où Buffy est attaquée par des vampires. Cette scène pourrait paraître banale dans la série mais l'image d'ouverture - un gros plan sur la bouche d'une jeune fille hurlant et un zoom arrière qui laisse découvrir son visage - s'inscrit dans un décalage burlesque et une exagération 
qui laissent entendre la dimension comique de la scène. Buffy ne parvient pas à se tirer de ce traquenard mais elle est sauvée in extremis par Xander - situation qui paraît encore possible bien que surprenante à ce stade de la série, où Xander n'est pas encore considéré comme un combattant efficace. Xander se débarrasse rapidement de l'ennemi avant de demander à Buffy si elle va bien, d'une voix sourde de héros séducteur. Il monte sur scène, empoigne sa guitare, sous les yeux de l'hérö̈ne qui le contemple forcément avec amour (voir figure 2).

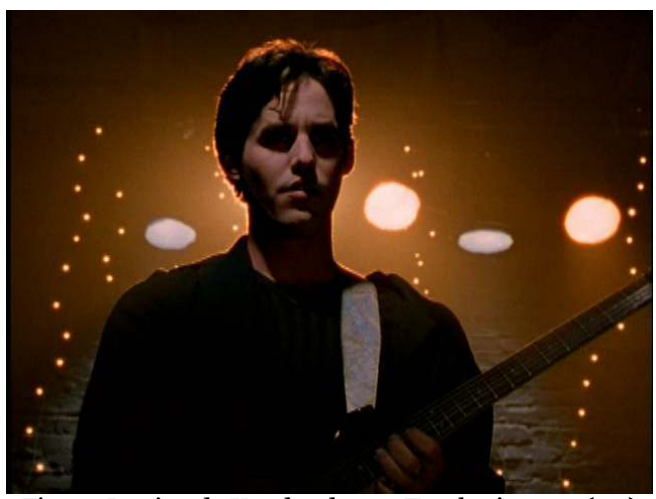

Fig. 2 : Le rêve de Xander dans « Teacher's pet » (1.4)

Il s'agit bien sûr d'un rêve et Xander est rappelé à la réalité - son cours de biologie durant lequel il s'était assoupi. Le prégénérique joue de sa nature même de commencement en utilisant le fait que le téléspectateur ne peut pas identifier le statut de l'image qu'il voit dans la mesure où aucune autre information ne lui a encore été donnée et qu'il est donc amené à accepter la première scène comme étant $a$ priori la réalité. Même si des indices lui sont fournis, qui indiquent que la scène n'est pas une scène habituelle, il n'en connaît pas encore le statut. En effet, dans Buffy, à la question du fantastique s'ajoute la question de l'humour : puisque certains épisodes sont extrêmement burlesques, le spectateur est prêt à envisager et à accepter presque tous types d'intrigues.

Cette stratégie du prégénérique permet aux auteurs de mettre en place, dans cet exemple, un tableau particulièrement comique mais aussi, par une voie détournée et expressive, de poser une problématique essentielle du personnage de Xander - son désir d'être un héros. Il est l'humain du groupe, celui qui, en ce tout début de série, ne semble pas encore y trouver sa place et son utilité. Comme nous l'avons dit plus haut, Buffy est avant tout une série fondée sur les personnages, leur évolution et les relations qui se tissent entre eux, et 
c'est pourquoi le prégénérique peut se concentrer moins sur les événements et l'intrigue que sur la psychologie du personnage qui en sera le moteur principal. Dans notre exemple, l'épisode va ensuite mettre en scène une femme, professeur de biologie remplaçante, qui séduit Xander, mais se révèle être une mante religieuse. La séquence d'ouverture annonce donc bien le sujet de l'épisode (Xander et la séduction érotique) mais elle le fait non pas en termes d'intrigues mais d'approfondissement du personnage, de ses angoisses et fantasmes.

\section{Quelle est la longueur du prégénérique ?}

Si dans certaines séries ou dans certains épisodes, la séquence d'ouverture peut être très courte, dans d'autres, elle dure plusieurs minutes. Les exemples de teasers de cinq à dix minutes sont nombreux. Dans les deux cas, le statut de cette séquence varie considérablement: de simple scénette d'ouverture, la séquence prégénérique peut aussi bien constituer un acte entier, comme dans Alias (ABC, 2001-2006). Ainsi la séquence prégénérique de l'épisode 1.4 dure-t-il plus de huit minutes. Elle consiste en la suite exacte de la fin de l'épisode précédent, puisque celui-ci se terminait sur un cliffhanger : Sidney ouvre, avec une autre agent ennemi, une valise et semble effrayée par le contenu qui reste invisible pour le spectateur. L'épisode 4 commence sur ces mêmes images, révélant par la suite que la valise contient une bombe. L'ensemble de la séquence porte sur la confrontation de Sidney avec cette ennemie. En effet, plus qu'il ne commence ce nouvel épisode, le teaser termine l'épisode précédent, non pas de manière anecdotique mais bien par une séquence qui constitue presque le quart de l'épisode. Dès lors, il ne s'agit plus seulement pour le prégénérique d'ouvrir l'épisode, mais bien d'en constituer une partie aussi conséquente que les autres actes. La question de l'isolement de la séquence se pose ici de manière plus problématique dans la mesure où elle joue presque à armes égales avec les autres parties de l'épisode. Ainsi, le statut de la séquence prégénérique se conçoit-il en fonction de la distribution des données narratives : quel est exactement l'apport du teaser dans l'ensemble de l'épisode ou de la série?

\section{Jouer avec les codes : entre rupture et continuité}

Au-delà des différentes fonctions narratives que nous venons d'évoquer, le prégénérique endosse également des fonctions esthétiques ainsi que des fonctions d'identification générique de l'épisode et de la série. Les différentes stratégies de la typologie qui précède se mêlent à d'autres jeux, métatextuels, inter- ou intratextuels, qui complexifient davantage encore la séquence prégénérique. Celle-ci inscrit l'épisode qui va suivre dans un certain genre, dans un ton et une esthétique spécifiques, mais elle rattache aussi ce même épisode à 
l'identité narrative et visuelle de la série dans son ensemble. Elle est un seuil qui permet au téléspectateur de reconnaître immédiatement la série qu'il regarde. La séquence prégénérique est, par conséquent, essentiellement fondée sur un jeu de variations à partir de données fixes.

\section{Le prégénérique du pilote et le recyclage des codes}

Dans l'analyse de la sérialité, le cas de la séquence prégénérique du pilote constitue bien sûr un cas particulier, au sens où rien ne le précède mais que tout découle de lui. Cette séquence est, par bien des aspects, une matrice, c'est-à-dire un modèle, mais aussi une ouverture sur les possibles que déclineront ensuite les prégénériques des autres épisodes. Elle peut d'ores et déjà mettre en place une formule et se situer par rapport aux codes génériques ou, au contraire, se poser en hapax pour entrer dans l'univers fictionnel sans pour autant en dévoiler tous les secrets.

À propos des séries fantastiques, nous avons déjà évoqué l’idée selon laquelle le teaser est souvent l'occasion de présenter une première victime d'un fait surnaturel: il s'inscrit ainsi dans une codification du genre avec laquelle la série va pouvoir jouer. Le cas du teaser du pilote de Buffy the Vampire Slayer est tout à fait remarquable car non seulement il installe un schéma qui sera celui des épisodes suivants mais il met également en place les éléments qui distinguent Buffy d'autres séries fantastiques, en opérant un renversement des attentes du téléspectateur. Le prégénérique y joue en effet des codes des séries fantastiques par son usage de repères spatiotemporels spécifiques, par l'ambiance inquiétante et par la musique qui souligne le suspense. Le spectateur sait qu'une menace pèse mais, puisque le jeune homme semble pousser la jeune fille à le suivre, il pense que c'est elle qui sera la victime. Or, cette jeune fille n'est autre que Darla, très vieux vampire qui assassine le garçon avant que le générique ne commence. Ainsi, la jeune fille blonde, qui paraît sans défense et qui serait une victime désignée dans n'importe quel film d'horreur, révèle-t-elle son identité de prédateur et sa force sous son apparence inoffensive. Bien sûr, le personnage même de Buffy relève de ce schéma puisqu'elle aussi est une adolescente blonde qui endosse cette fois le rôle de super-héroïne. Le prégénérique inscrit par conséquent la série dans les codes du genre tout en les subvertissant, et anticipe sur ce qui sera un principe fondateur de l'ensemble de la série. Enfin, alors qu'on pense qu'il présente des personnages tout à fait secondaires (les malheureuses victimes que l'on ne revoit jamais ensuite), ce teaser met en fait en scène un personnage qui s'avèrera récurrent, le vampire Darla.

True Blood (HBO, 2008-), dans les années 2000, joue du même procédé. Autre série sur des vampires, elle renverse, dans sa première séquence d'ouverture, l'ensemble des codes et des attentes du 
spectateur. Elle met en scène un couple de jeunes qui possèdent toutes les caractéristiques des victimes potentielles mais qui sont finalement épargnés. Par ailleurs, le vampire de la séquence n'est précisément pas celui que l'on croit. Alors que le propriétaire du drugstore avec ses tatouages, sa bague en forme de crâne et son bracelet de munitions, laisse augurer un danger - d'autant qu'il parle de vampires en disant " nous »-, il apparaît qu'il est humain et ne fait que plaisanter. Et c'est le client banal qui est en fait un vampire (voir figure 3).

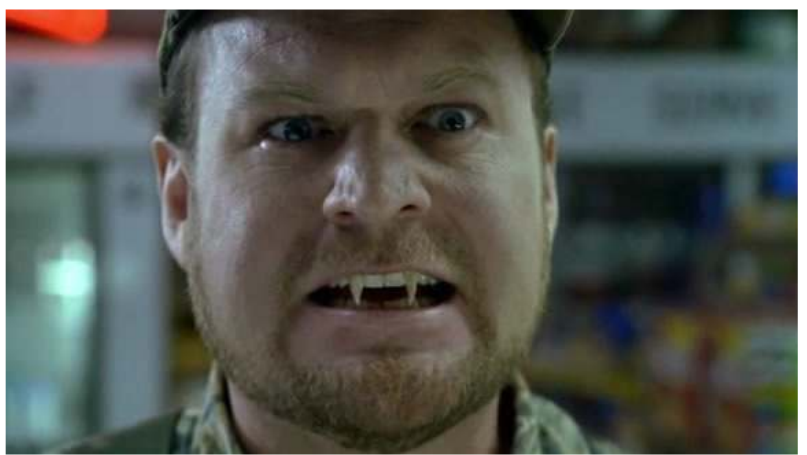

Fig. 3 : Première séquence du pilote de True Blood

La séquence joue donc sur les codes de représentation des personnages pour déjouer leur identification. Mais elle se poursuit avec humour puisque le vampire ne fait aucune victime et clôt la scène avec un sourire qui fait ressortir ses canines, en lançant un joyeux « Have a nice day ». Le prégénérique joue donc sur les contrastes et sur la déconstruction humoristique des codes du genre. Les vampires de Louisiane n'y ont rien de commun avec la tradition des dandys des romans d'Anne Rice mais peuvent tout aussi bien être des Américains moyens qui cherchent à se faire une place au sein de la société. Il s'agit donc bien d'installer l'identité de la série en la caractérisant par rapport au corpus préexistant et aux codes génériques. Comme déjà avec Buffy, mais ce sera le cas de manière plus évidente encore dans les séries des années 2000, nous sommes entrés, au tournant du siècle, dans une ère référentielle où les séries se situent de plus en plus explicitement vis-àvis de ce qui précède, citent ouvertement leurs modèles, les subvertissent ou les parodient avant de faire de même avec leurs propres codes, une fois le modèle installé au cours des saisons. 


\section{Le prégénérique du pilote et l'instauration de codes esthétiques}

La séquence prégénérique d'une série impose aussi ses particularités propres en termes de choix esthétiques. Ainsi, The $X$ Files, du fait de son exigence visuelle et de la mise en place de motifs récurrents en termes de mise en scène, impose-t-elle de nouveaux standards de qualité et de structuration, en se rapprochant du cinéma, ce que revendique d'ailleurs son créateur Chris Carter ${ }^{12}$. Par exemple, la série s'ouvre sans véritable prégénérique puisque l'épisode 1 ne comporte pas de générique, comme c'était souvent le cas des pilotes dans les années 1990. Pourtant, les crédits apparaissent après la première séquence, en surimpression sur l'image, comme à la suite d'un générique dans un épisode normal. De plus, comme dans le reste de la série, cette première séquence, qui est quand même isolée formellement de la suite, expose l'affaire sur laquelle les agents vont enquêter, tandis que le corps de l'épisode débute par l'entrée en scène des personnages principaux de la série, en l'occurrence Scully. Sans véritable coupure, le pilote présente donc une séquence qui correspond à tous les critères du prégénérique.

Cette séquence est une scène de nuit tournée dans une forêt où une jeune fille court en chemise de nuit ; une lumière apparait derrière les arbres, un jeune homme arrive à cet endroit et tous deux sont pris dans cette lumière. L'ancrage spatio-temporel - la forêt, la nuit - est un motif central de la série et l'approche réaliste de la mise en scène choisit d'ancrer le fantastique dans le monde le plus contemporain et le plus quotidien qui soit. Le pilote s'ouvre d'ailleurs sur une mention qui n'apparaîtra plus par la suite, indiquant que les événements racontés dans l'épisode sont inspirés de faits réels. La deuxième scène de cette séquence présente le corps de la jeune fille entouré de policiers sur ce qui semble bien être une scène de crime. Le lieu de l'action s'affiche à l'écran comme s'il était tapé à la machine à écrire, autre particularité de l'esthétique réaliste de la série À cela s'ajoute la musique électronique de Mark Snow qui travaille peu sur des mélodies et compose davantage des ambiances inquiétantes qui contribuent largement à l'esthétique de la série. Nombreux sont donc les repères esthétiques qui définissent l'identité de la série et qui sont mis en place dès cette séquence. Or, une fois cette identité posée, la séquence prégénérique des épisodes suivants devient un moment de rappel de ces principes.

Le teaser, qui est habituellement le lieu de l'identification de la série et de la reconnaissance de ses codes de fonctionnement, peut cependant devenir, l'espace d'un épisode, le lieu de la surprise, du

${ }_{12}$ Ce rapport au cinéma dans le discours des showrunners est fréquente au début des années 1990. On peut penser à Twin Peaks (ABC, 1990-1991) ou à E.R. (NBC, 1994-2009). 
décalage, de la référence intertextuelle, du commentaire méta-narratif ou de tout autre procédé qui isole l'épisode.

\section{Des prégénériques marqués}

Un dernier critère pourrait donc s'ajouter à la typologie dressée précédemment, qui permettrait de dire si le prégénérique possède une esthétique propre, se démarquant de l'ensemble de la série (prégénérique marqué) ou si, au contraire, posant les jalons de l'esthétique de la série, il s'y intègre harmonieusement (prégénérique non marqué)? Cette dimension est aussi un indicateur pour le téléspectateur qui peut alors sentir, dès les premières images d'un épisode, que celui-ci sera spécial. C'est le cas de l'épisode musical de Buffy, "Once more with feeling » (6.7). On aurait pu imaginer que le démon de la danse arrive en ville et que les numéros musicaux ne commencent que dans l'épisode proprement dit, mais l'effet est bien sûr décuplé par l'ouverture musicale dès le prégénérique, qui plonge immédiatement le spectateur dans l'ambiance d'un épisode qu'il sait être exceptionnel au sein d'une série qui n'est pas musicale.

Le cas de « The Post-modern Prometheus » (5.5) de The X-Files en est un autre exemple remarquable. L'épisode est en noir et blanc et utilise les codes du cinéma fantastique (voir figure 4), renvoyant notamment, comme son titre l'indique, à Frankenstein.

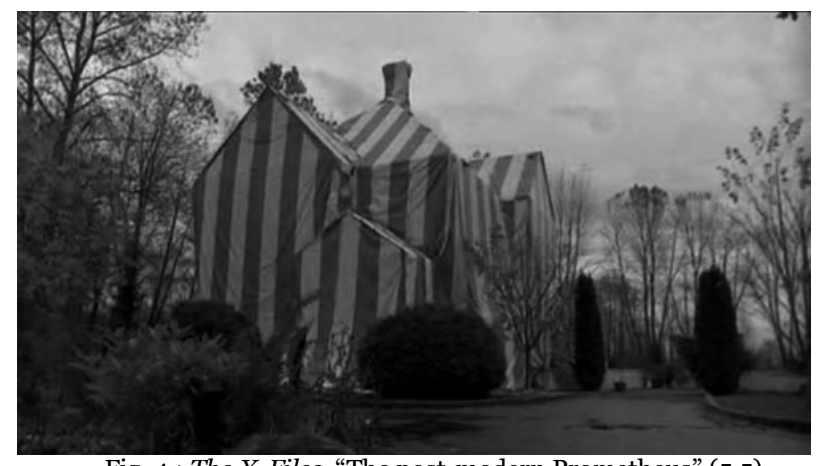

Fig. 4 : The X-Files, "The post-modern Prometheus" (5.5)

Mais il détourne aussi ces codes de manière burlesque puisqu'il met en scène un personnage monstrueux par son apparence mais inoffensif, dont la particularité, outre son physique, est d'être fan de la chanteuse Cher, ce que révèle le prégénérique particulièrement déroutant et incompréhensible au premier abord. Alors que, dans le teaser du pilote de Buffy évoqué plus haut, la série fonde ses propres codes en renversant ceux des œuvres qui l'ont précédée (la jeune fille superhéroïne plutôt que victime du vampire), The $X$-Files, à l'inverse, déjoue 
temporairement ses codes propres en se fondant sur une reprise de codes antérieurs, sur le mode à la fois de l'hommage et de la parodie ponctuels.

Les exemples de ce type sont nombreux. On peut penser à l'épisode 8 de la saison 5 de Supernatural, "Changing Channels ", dont la scène d'ouverture présente les personnages habituels de la série mais comme si Supernatural était une sitcom et non pas une série fantastique - en utilisant des rires enregistrés, un décor unique filmé toujours du même angle ainsi qu'une palette de couleurs très vives, contrastant radicalement avec l'esthétique de la série (voir figure 5).

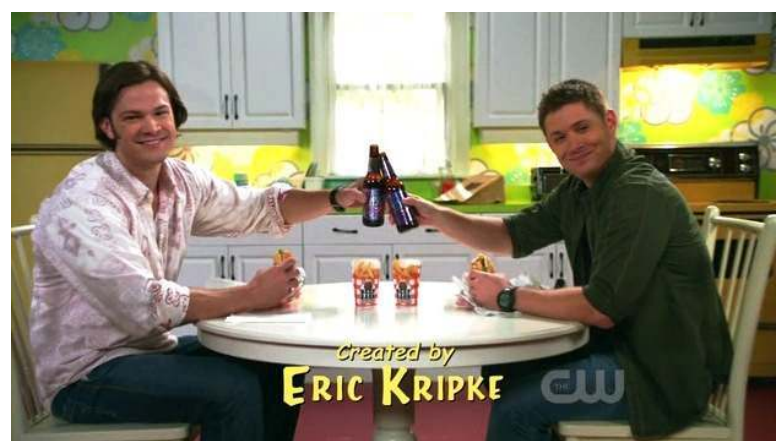

Fig. 5 : Générique de « Changing channels », Supernatural (5.8)

Le prégénérique est ici une scène qui se passe chronologiquement au milieu de l'histoire de l'épisode. Mais le choix de l'isoler au tout début de l'épisode crée un effet de contraste et de surprise redoublé parce que le spectateur l'aborde sans contexte et sans y être préparé.

Ainsi, la séquence prégénérique est un moment privilégié au sein de la narration sérielle et les auteurs s'en servent diversement depuis le début des années 1990, exploitant l'ensemble des possibilités de ce format, soit pour établir des normes, des procédés d'identification et de reconnaissance qui permettent au spectateur d'entrer progressivement dans un univers ou de le retrouver confortablement chaque semaine, soit pour ouvrir un espace de création autonome où les possibilités sont décuplées en raison de l'isolement relatif de cette section narrative. Espace du rêve, de la transgression, du comique, de la référence, de la réflexivité, la séquence prégénérique est le lieu d'une expérimentation potentielle et cristallise les enjeux de la narration sérielle par sa position cruciale et son ambivalence entre la continuité et la rupture au sein de l'épisode même, mais aussi plus largement au sein de la série. C'est un seuil qui fournit de manière directe ou indirecte des informations sur l'épisode à 
venir. Dans les séries fantastiques, elle permet souvent de frapper un grand coup dès le début de l'épisode, pour capter le téléspectateur, tout en interrogeant la nature véritable de l'événement ou de l'individu surnaturel qui est soumis à notre attention, soulignant ainsi le questionnement du rapport à la " réalité » posé par la série. En outre, la séquence prégénérique ne doit pas être pensée comme la première unité narrative d'une chaîne chronologique. Elle peut l'être mais ce n'est pas toujours le cas. Son véritable enjeu réside dans la manière dont elle met en valeur un aspect de l'épisode ou de la série en l'isolant. Point de suture tout autant que point de rupture, au sein de la série mais également au sein de l'épisode qu'elle ouvre, la séquence prégénérique peut jouer le rôle d'une petite poupée russe ou celui d'un jack in the box et acquiert ainsi un statut de "micro film » à l'ouverture de séries qu'elle alimente et grignote tout à la fois.

\section{Bibliographie}

Aubry Danielle, Du roman-feuilleton à la série télévisuelle. Pour une rhétorique du genre et de la sérialité, Berne, Peter Lang, 2006.

Benassi Stéphane, Séries et feuilletons T.V. Pour une typologie des fictions télévisuelles, Liège, Éditions du CEFAL, 2000.

Colonna Vincent, L’Art des séries télé, Paris, Éditions Payot et Rivages, 2010.

HUDELET Ariane, «Un cadavre ambulant, un petit-déjeuner sanglant, et le quartier Ouest de Baltimore : le générique, moment-clé des séries télévisées ", GRAAT On-Line, Anglophone Studies, No. 6, décembre 2009. http://www.graat.fr/backissuepiegesseriestv.html, lien consulté le 11 décembre 2014.

Roman James, From Daytime to Primetime, The History of American Television Programs, Westport, Greenwood Press, 2005.

Soulez Guillaume (éd.), Sérialité : densités et singularités, Mise au Point, No. 3, 2011. http://map.revues.org/75, lien consulté le 11 décembre 2012.

TYLSKI Alexandre, Le Générique de cinéma. Histoire et fonctions d'un fragment hybride, Toulouse, Presses Universitaires du Mirail, 2008.

VERAT Éric, Génériques ! Les séries américaines décryptées, Lyon, Les Moutons électriques, 2012. 


\section{L'auteure}

Claire Cornillon est ingénieur de recherche postdoctoral à l'université du Havre. Elle est agrégée de Lettres Modernes et docteur en Littérature

Comparée. Ces recherches les plus récentes portent sur les séries télévisées ainsi que sur le transmédia. Elle co-dirige le projet "Narrations sérielles et Transmédialité » à l'université Sorbonne Nouvelle-Paris 3 depuis 2012. Elle est par ailleurs directrice de la rédaction de Lintermede.com. 